\title{
Correction to: Bounds on Signless Laplacian Eigenvalues of Hamiltonian Graphs
}

\author{
Milica Anđelić ${ }^{1}$ (D) Tamara Koledin $^{2}$ (D) Zoran Stanić ${ }^{3}$
}

Published online: 30 January 2021

(c) Sociedade Brasileira de Matemática 2021

\section{Correction to: Bull Braz Math Soc, New Series https://doi.org/10.1007/s00574-020-00211-y}

In our original paper the incorrect labelling of the number of vertices of the line graph $L(G)$ in the proof of Theorem 2 has led to the inequality

$$
q_{n} \leq \frac{\overline{d^{2}}-4}{\bar{d}-2}-2-\sqrt{\left(\frac{\overline{d^{2}}-4}{\bar{d}-2}-6\right)^{2}+8(\bar{d}-2)},
$$

which does not necessarily hold. The size of $L(G)$ is $m$ and therefore its least eigenvalue should have been labelled as $\lambda_{m}(L(G))$ (not $\lambda_{n}(L(G))$ ). Below we indicate the necessary changes implied by this glitch.

- All the statements regarding the upper bound on the least signless Laplacian eigenvalue of Hamiltonian graphs should be removed. The statements regarding the signless Laplacian index of Hamiltonian graphs remain.

The original article can be found online at https://doi.org/10.1007/s00574-020-00211-y.

$\triangle$ Milica Anđelić

milica.andelic@ku.edu.kw

Tamara Koledin

tamara@etf.rs

Zoran Stanić

zstanic@math.rs

1 Department of Mathematics, Kuwait University, Safat 13060, Kuwait

2 Faculty of Electrical Engineering, University of Belgrade, Bulevar kralja Aleksandra 73, 11000 Belgrade, Serbia

3 Faculty of Mathematics, University of Belgrade, Studentski trg 16, 11000 Belgrade, Serbia 
- Example 2 and 3 should be modified accordingly. Non-Hamiltonicity is now proved only using the inequality (4) from Corollary 3.

The original article has been updated.

Publisher's Note Springer Nature remains neutral with regard to jurisdictional claims in published maps and institutional affiliations. 